\title{
Immunohistochemical characterization of intestinal neoplasia in zebrafish Danio rerio indicates epithelial origin
}

\author{
Colleen E. Paquette ${ }^{1,4}$, Michael L. Kent ${ }^{1}$, Tracy S. Peterson ${ }^{1,5}$, Rong Wang ${ }^{2}$, \\ Roderick H. Dashwood ${ }^{2,6}$, Christiane V. Löhr ${ }^{3, *}$ \\ ${ }^{1}$ Department of Microbiology, Oregon State University, Corvallis, Oregon, USA \\ ${ }^{2}$ Linus Pauling Institute, Oregon State University, Corvallis, Oregon, USA \\ ${ }^{3}$ Department of Biomedical Sciences, College of Veterinary Medicine, Oregon State University, Corvallis, Oregon, USA \\ ${ }^{4}$ Present address: Translational Imaging Center, University of Southern California, Los Angeles, California, USA \\ ${ }^{5}$ Present address: University of Arkansas Regulatory Science Center, Pine Bluff, Arkansas, USA \\ ${ }^{6}$ Present address: Center for Epigenetics \& Disease Prevention, Texas A\&M Health Science Center, Houston, Texas, USA
}

\begin{abstract}
Spontaneous neoplasia of the intestinal tract in sentinel and moribund zebrafish Danio rerio is common in some zebrafish facilities. We previously classified these tumors as adenocarcinoma, small-cell carcinoma, or carcinoma otherwise unspecified based on histomorphologic characteristics. Based on histological presentation, the primary differential diagnosis for the intestinal carcinomas was tumor of neuroendocrine cells (e.g. carcinoids). To further characterize the phenotype of the neoplastic cells, select tissue sections were stained with a panel of antibodies directed toward human epithelial (cytokeratin wide spectrum screening [WSS], AE1/AE3) or neuroendocrine (S100, chromogranin A) markers. We also investigated antibody specificity by Western blot analysis, using a human cell line and zebrafish tissues. Nine of the intestinal neoplasms (64\%) stained for AE1/AE3; 7 (50\%) also stained for WSS. None of the intestinal neoplastic cells stained for chromogranin A or S100. Endocrine cells of the pituitary gland and neurons and axons of peripheral nerves and ganglia stained for chromogranin A, whereas perineural and periaxonal cells of peripheral intestinal ganglia, and glial and ependymal cells of the brain stained for S100. Immunohistochemistry for cytokeratins confirmed the majority of intestinal neoplasms in this cohort of zebrafish as carcinomas.
\end{abstract}

KEY WORDS: Zebrafish $\cdot$ Neoplasia $\cdot$ Intestine $\cdot$ Carcinoma $\cdot$ Immunohistochemistry $\cdot$ Cytokeratin Western blot

\section{INTRODUCTION}

For over a decade, spontaneous intestinal neoplasia has been observed in zebrafish Danio rerio submitted to the ZIRC (Zebrafish International Resource Center) diagnostic service (Spitsbergen et al. 2012, Paquette et al. 2013). Many of the fish from these populations also displayed preneoplastic changes in the intestine, including epithelial hyperplasia and dysplasia. Based on routine histology, neoplastic lesions were classified either as adenocarcinoma $(50.4 \%)$ or small cell carcinoma $(37.2 \%)$, or other (carcinoma not otherwise specified, tubular adenoma, tubulovillous adenoma, 12.4\%) (Paquette et al. 2013).

The cellular phenotype of these neoplasms has not been fully elucidated. Considering the location and morphology, the primary alternative to intestinal car- 
cinomas are small cell carcinoma or carcinoid tumors derived from neuroendocrine cells (Klöppel \& Anlauf 2005, Modlin et al. 2008). The purpose of this study was to more fully characterize zebrafish intestinal tumors using immunohistochemistry. There is close conservation between human and zebrafish tumorigenic mechanisms at the molecular, cellular, and tissue levels, including expression of tumor antigen target epitopes (Amatruda \& Patton 2008, Liu \& Leach 2011). Based upon interspecies target epitope conservation and the limited availability of zebrafish-specific antibodies, we chose to evaluate tumor antigen expression using antibodies raised against human antigens. Both wide spectrum screening (WSS) and AE1/AE3 are cytokeratin markers expressed in human intestinal adenocarcinomas (Chu \& Weiss 2002). S100 is a marker for neural crest-derived-cells found in human neurogenic neoplasms (e.g. schwannoma, melanoma, ependymoma, astroglioma) and gastrointestinal stromal tumors (GISTs) (Miettinen \& Lasota 2001). Chromogranin A is expressed in chromaffin cells of endocrine and neuroendocrine origin and their respective neoplasms, such as pheochromocytoma and small-cell carcinomas (Ferrari et al. 1999). In addition, WSS and AE1/AE3 cytokeratins, S100 and chromogranin A antibodies were evaluated by Western blotting, in order to compare and contrast differences, if any, between the respective human and zebrafish proteins.

\section{MATERIALS AND METHODS}

\section{Cases}

A subset of paraffin-embedded sentinel and moribund zebrafish, previously fixed with Dietrich's, was selected from the archive at the ZIRC diagnostic service, based upon the presence of intestinal tumors previously classified as adenocarcinoma, small-cell carcinoma, or carcinoma not otherwise specified (Paquette et al. 2013). These specimens demonstrated distinct histomorphologic characteristics of the comparative mammalian tumors (Lingeman \& Garner 1972, Sidhu 1979, Brenner et al. 2004), determined using routine hematoxylin and eosin-stained slides.

\section{Western blotting}

Five whole frozen zebrafish $\sim 30 \mathrm{~d}$ post fertilization (dpf) were processed for immunoblot analyses. The animals were pooled and homogenized with IP lysis buffer (Roche) and incubated overnight at $4^{\circ} \mathrm{C}$. Protein concentration was determined using the Thermo Scientific Pierce Micro BCA protein assay (Thermo Scientific), with bovine serum albumin as standard. Zebrafish protein samples were then prepared and separated by electrophoresis on a SDS-PAGE gel using MagicMark ${ }^{\mathrm{TM}}$ XP Western Protein Standard (Life Technologies) and human acute monocytic leukemia cell line HTP-1 (ATCC ${ }^{\circledR}$ TIB-202 ${ }^{\mathrm{TM}}$ American Type Culture Collection) as positive controls. Proteins were transferred onto a nitrocellulose membrane (Life Technologies,) and blocked by immersion in $2 \%$ bovine serum albumin (BSA) dissolved in phosphate-buffered saline (PBS). The membranes were then incubated at $4^{\circ} \mathrm{C}$ overnight with the primary antibodies against WSS (Dako Z0622) 1:2000, AE1/AE3 (Dako M3515) 1:400, S100 (Dako Z0311) 1:500 or chromogranin A (Dako A0430) 1:2000 (Dako, Agilent Technologies). After incubation, the membranes were washed with PBS containing $0.1 \%$ Tween-20 6 times and incubated for $1 \mathrm{~h}$ with a secondary antibody of goat anti-rabbit IgG-HRP (WSS, S100, and chromogranin A) or goat anti-mouse IgGHRP (AE1/AE3) (Dako, Agilent Technologies). Membranes were subsequently washed 3 times with PBS containing $0.1 \%$ Tween-20. Substrate development for photo documentation was performed using the Pierce $^{\mathrm{TM}}$ ECL chemiluminescent substrate (Thermo Scientific).

\section{Immunohistochemistry}

Immunohistochemistry was performed according to standard operating procedures of the Veterinary Diagnostic Laboratory at Oregon State University. In brief, 4 to $5 \mu \mathrm{m}$ sections on charged slides (Tanner Scientific) were rehydrated. Slides were either high temperature antigen retrieved (chromogranin A: Dako A0430 [1:1000]; S100: Dako Z0311 [1:400]) in a microwave pressure cooker for 10 min or enzymatically digested with Proteinase K (Dako S3020) for 5 min (CK(WSS): Dako Z0622 [1:500]; AE1/AE3: Dako M3515 [1:100]). In a Dako Autostainer primary antibodies were applied for $30 \mathrm{~min}$ at room temperature followed by MaxPoly-One polymer HRP rabbit or mouse (MaxVision Biosciences) for $10 \mathrm{~min}$ at room temperature. Signal was developed with chromogen Nova Red (SK-4800, Vector Laboratories), and slides were counterstained with Dako hematoxylin (s3302). Sections of whole zebrafish served as positive and negative controls for immunohistochemistry. 
Considering the subjectivity in evaluation of immunohistochemistry results, the slides were read independently by 3 of the authors (C. E. Paquette, M. L. Kent, and C. V. Löhr). Results were recorded as follows for each antibody: positive, equivocal (weak staining or few neoplastic cells showing positivity), or negative.

\section{RESULTS}

\section{Cases}

The fish selected for analysis were sourced from 6 individual facilities and included 7 females and 7 males, ranging in age from 301 to $1071 \mathrm{dpf}$. Both wild-type and mutant lines were represented. Six tumors were located in the anterior third of the intestine, 7 were mid-intestine, and 1 was located in the mid-distal intestine. Four of the tumors analyzed were classified as adenocarcinoma, 5 as small-cell carcinoma, and 5 as carcinoma not otherwise specified.

\section{Western blotting}

In proteins from whole zebrafish homogenate, WSS stained 2 distinct bands at $\sim 40 \mathrm{kDa}$, while a series of bands ranging from 48 to $59 \mathrm{kDa}$ was expected and observed with the human cell homogenate (Adem et al. 2002, Chu \& Weiss 2002) (Fig. 1a). AE1/AE3 recognized several zebrafish protein bands between $\sim 38$ and $41 \mathrm{kDa}$, whereas a series of bands at $\sim 40$ and between 48 and $67 \mathrm{kDa}$ was expected and observed with the human cells (Woodcock-Mitchell et al. 1982, Chu \& Weiss 2002) (Fig. 1b). S100 reacted with a zebrafish protein at $\sim 10 \mathrm{kDa}$, compared to $\sim 21 \mathrm{kDa}$ for human S100 protein (Singh \& Cheng 1996) (Fig. 1C). Chromogranin A recognized a band at $\sim 44 \mathrm{kDa}$ in the zebrafish tissue and at the expected $74 \mathrm{kDa}$ in the human cells (Montero-Hadjadje et al. 2008) (Fig. 1d).

\section{Immunohistochemistry}

The expression of WSS, AE1/AE3, S100, and chromogranin A antibodies was analyzed in normal tissue and tissue from intestinal tumors from 14 individual zebrafish. Intestinal tumors included adenocarcinoma (Fig. 2a,c), small-cell carcinoma (Fig. 2b,d), and carcinoma not otherwise specified (not shown).

Strong staining for WSS was present in normal epithelial cells of the intestine (Fig. 3a), skin, nares,

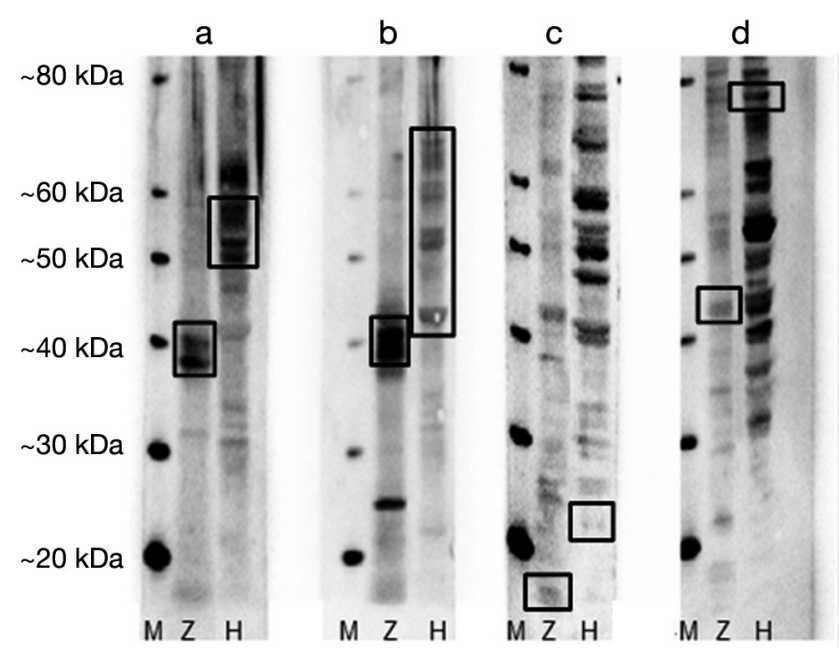

Fig. 1. Western blot against (a) wide spectrum screening (WSS), (b) AE1/AE3, (c) S100, and (d) chromogranin A compared to expected targeted protein sizes (indicated by boxes). M: marker protein; Z: normal whole adult zebrafish tissue homogenate; H: HTP-1 cells (human)

and gills (Fig. 3b), and renal collecting ducts of adult zebrafish, serosal cells of the coelomic cavity and meninges, and cross-reacted with chondrocytes and endothelium. Neural tissue and exocrine pancreas were negative. AE1/AE3 demonstrated the same staining pattern as WSS including staining of epithelial cells of the intestine and gills (Fig. 3c and Fig. 3d, respectively) and no staining of nervous tissue and exocrine pancreas. However, WSS produced a stronger staining reaction in epithelial cells and less intense cross-reactivity in endothelial cells than AE1/AE3. Seven of the $14(50 \%)$ intestinal neoplasms scored positive for WSS (Fig. 3e), and 9 of the $14(64 \%)$ were positive for AE1/AE3 (Fig. 3f) (Table 1).

Chromogranin A reacted positively with scattered neurons of vertebral ganglia, most cells in the pituitary gland, some nerve fibers in the normal brain and spinal cord, axons and/or sheath cells in peripheral nerves, and thin fibers in the lateral line, sensory organs of the skin and along the cutaneous basement membrane, skeletal muscle, and myenteric plexus (Fig. 3g). Staining intensity was stronger for the latter. Normal intestinal epithelium was negative. All of the intestinal tumors were regarded as negative for chromogranin A (Fig. 3g).

In normal zebrafish tissue, S100 antibody showed strong immunoreactivity with glial cells in the nervous tissue including vertebral and myenteric ganglia (Fig. 3h), the nasal epithelium, meninges, thin fibers in the lateral line, skeletal musculature, and indi- 

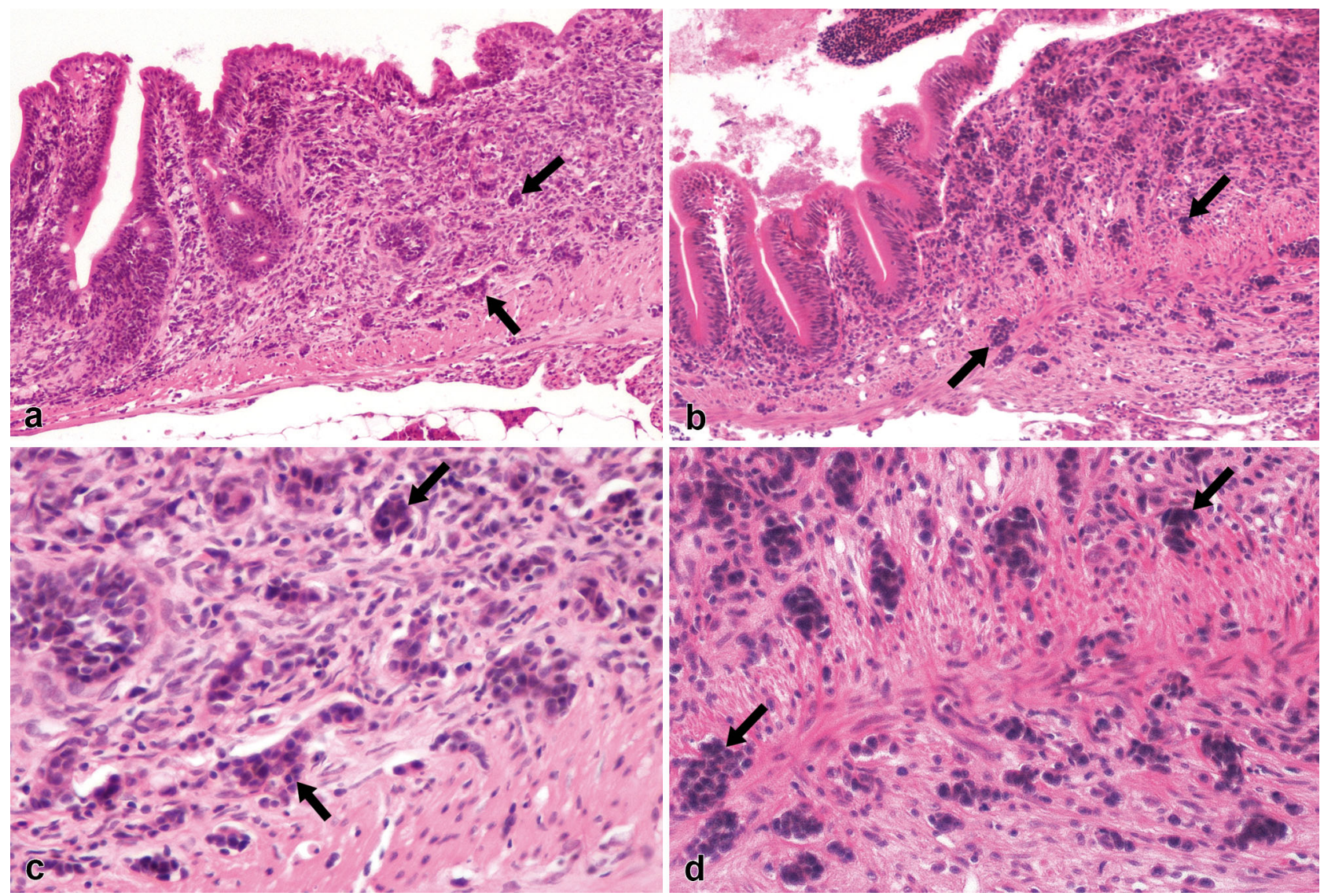

Fig. 2. Danio rerio. Intestine with neoplasia from 2 out of 14 individuals (see Table 1) . (a) Fish 1. Intestinal adenocarcinoma with neoplastic cells (arrows) within the lamina propria and muscularis, extending to the serosal layer. Hematoxylin and eosin (H\&E). (b) Fish 7. Intestinal small cell carcinoma infiltrating the lamina propria with invasion into the muscularis (arrows). H\&E. (c) Fish 1. Higher magnification of the intestinal adenocarcinoma in (a) shows neoplastic cells forming solid and pseudoacinar structures. (d) Fish 7. Higher magnification of intestinal small cell carcinoma in (b) shows fusiform neoplastic cells forming small aggregated nests

vidual cells in occasional renal tubules and showed weak reactivity in endocrine cells of the pituitary gland. Normal intestinal epithelium was negative for S100. All intestinal tumors were scored negative for S100 (Fig. 3h), except for 2 carcinomas designated 'equivocal' by 2 of the evaluators.

\section{DISCUSSION}

We recently reported, in a retrospective survey of the ZIRC diagnostic database, on the occurrence of intestinal tumors among zebrafish from several laboratories (Paquette et al. 2013). Some laboratories exhibited a high prevalence, and the majority of the intestinal tumors within that study were classified as adenocarcinomas, small-cell carcinomas, or carcinomas otherwise unspecified based upon histomor- phology. Immunohistochemical analysis reported here indicates that most, if not all, of the neoplasms are of epithelial origin. Two-thirds of the intestinal zebrafish tumors were positive for cytokeratins, while none stained strongly positive with neural tissue markers. Neoplastic cells in the small cell carcinomas were more often negative for the 2 epithelial antibodies. These cells are morphologically less differentiated, with a small nucleus and minimal cytoplasm.

It is not surprising that not all intestinal carcinomas stained for cytokeratins. Poor differentiation and progression towards anaplasia or tumor formation from pluripotent blast cells (Kapoor \& Khanna 2004) is associated with expression patterns of intermediate forms that are untypical for a particular cell type. Stratification of expression can be observed even amongst neoplastic cells within the same tumor (Chu 


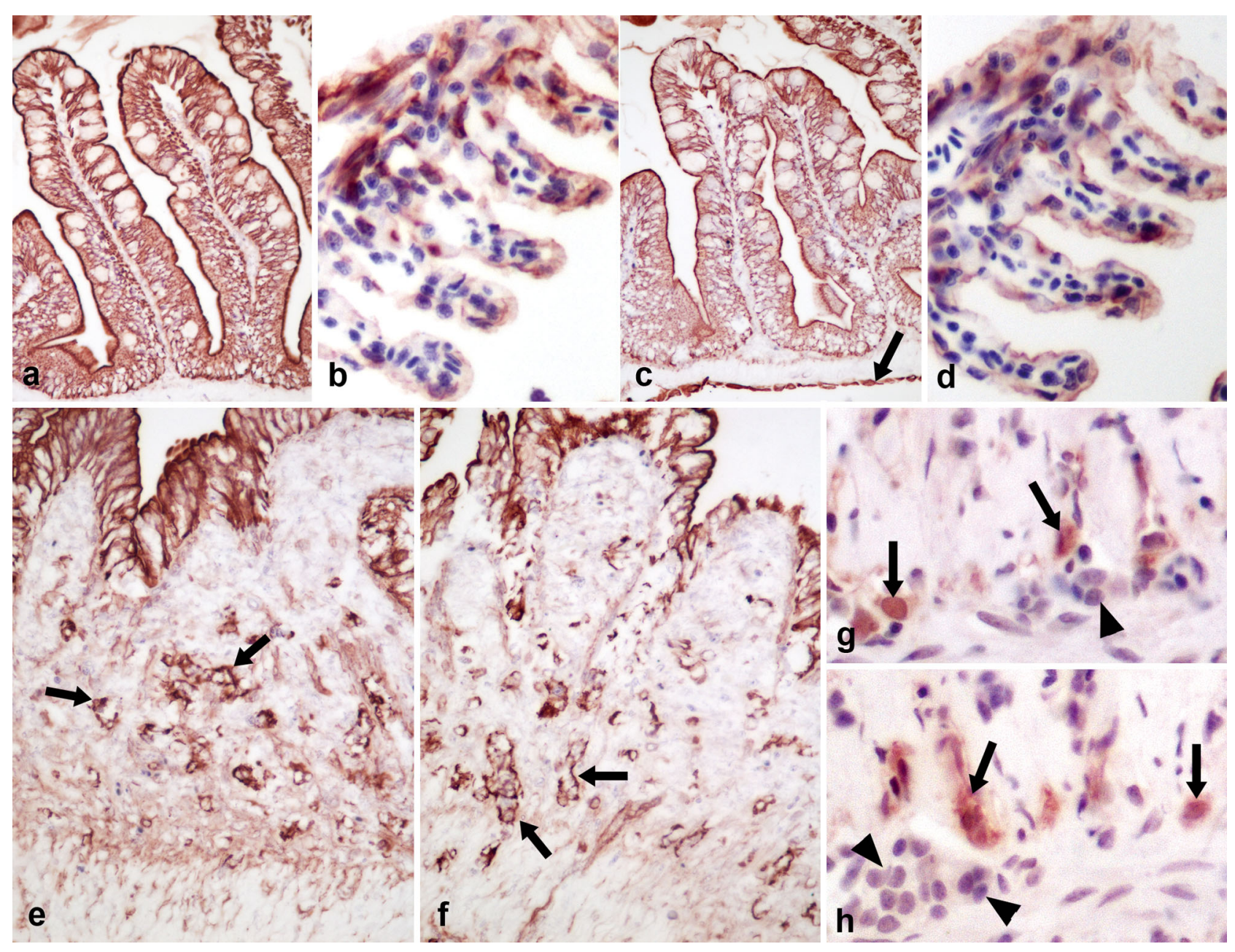

Fig. 3. Danio rerio. Immunohistochemistry of intestinal neoplasia and normal structures. All micrographs are from one individual (Fish 1, see Table 1) (a) Cytokeratin expression in the normal cells of the intestinal epithelium. Wide spectrum screening (WSS). (b) Cytokeratin expression in the gill epithelium. WSS. (c) Cytokeratin expression in the normal cells of the intestinal epithelium including mesothelial cells (arrow). AE1/AE3. (d) Cytokeratin expression in the gill epithelium. AE1/AE3. (e) Neoplastic cells (arrows) of the invasive intestinal tumor shown in Fig. 2a and previously classified as adenocarcinoma based upon histomorphologic characteristics stain for cytokeratin. WSS. (f) Neoplastic cells (arrows) of the same intestinal tumor shown in (e), stained for cytokeratin. AE1/AE3. (g) Neurons of myenteric plexus are stained (arrows), whereas neoplastic cells of the adenocarcinoma shown in Fig. 2a do not stain (arrowhead). Chromogranin A. (h) Normal ganglion cells of autonomic ganglia expressing positive staining (arrows), whereas neoplastic cells of the intestinal tumor shown in Fig. 2a do not stain (arrowheads). S100

\& Weiss 2002) and may be required for critical steps in tumor progression such as cell invasion (Gabbert et al. 1985). Specific protein bands for WSS and AE1/AE3 were detected in the prepared homogenates of adult zebrafish and human HTP-1 cells, albeit 11 to $16 \mathrm{kDa}$ below their predicted molecular weights in zebrafish tissue. AE1 and AE3 have been previously characterized by complimentary keratin blot-binding analysis (Conrad et al. 1998) and S100 by Western blot (Germanà et al. 2007). The small size of zebrafish allows for preparing one histologic slide containing all representative tissues from entire organ systems. This provides an excellent format for positive and negative controls for immunohistochemistry, as appropriate normal tissues are present in the exact specimen as the tissue of interest. In our study, a wide variety of epithelial cells were strongly positive with both cytokeratin stains.

Cells of gut-derived neuroendocrine neoplasms in vertebrates often stain for S100 and chromogranin A, particularly with the latter marker (Bunton 1994, Ferrari et al. 1999, Jirásek \& Mandys 2003, Modlin et al. 2008, Giandomenico 2010). None of the tumors examined here had convincing staining for either of the 
Table 1. Summary of wide spectrum screening (WSS), AE1/ AE3, S100, and chromogranin A staining of intestinal tumors in zebrafish submitted to the Zebrafish International Resource Center diagnostic service 2001-2011. In each column, the 3 symbols represent readings performed by 3 independent evaluators; + (positive), +/- (equivocal), - (negative). F: fish number; NOS: carcinoma not otherwise specified

\begin{tabular}{|c|c|c|c|c|c|c|c|c|c|c|c|c|}
\hline & \multicolumn{3}{|c|}{ WSS } & \multicolumn{3}{|c|}{$\begin{array}{l}\text { AE1/ } \\
\text { AE3 }\end{array}$} & \multicolumn{3}{|c|}{ S100 } & \multicolumn{3}{|c|}{$\begin{array}{l}\text { Chromo- } \\
\text { granin A }\end{array}$} \\
\hline \multicolumn{13}{|c|}{ Adenocarcinoma } \\
\hline $\mathrm{F} 1$ & + & + & + & + & + & + & - & - & - & - & - & - \\
\hline $\mathrm{F} 2$ & + & + & + & + & + & + & - & - & - & - & - & - \\
\hline F3 & - & - & - & - & - & - & - & $+/-$ & - & - & - & - \\
\hline $\mathrm{F} 4$ & - & - & - & - & - & - & - & - & - & - & - & - \\
\hline \multicolumn{13}{|c|}{ Small-cell carcinoma } \\
\hline F5 & - & - & - & $+/-$ & $+/-$ & $+/-$ & - & - & - & - & - & - \\
\hline F6 & - & - & - & $+/-$ & $+/-$ & $+/-$ & - & - & - & - & - & - \\
\hline F7 & - & - & - & $+/-$ & - & - & - & - & - & - & - & - \\
\hline F8 & + & + & + & + & + & + & - & - & - & - & - & - \\
\hline F9 & - & - & $+/-$ & + & + & + & - & - & - & - & - & - \\
\hline \multicolumn{13}{|c|}{ Carcinoma NOC } \\
\hline F10 & + & + & + & + & + & + & - & - & - & - & - & - \\
\hline F11 & - & - & - & + & $+/-$ & + & - & - & - & - & - & - \\
\hline F12 & + & $+/-$ & + & + & + & + & - & - & - & - & - & - \\
\hline F13 & + & + & + & + & + & + & $+/-$ & - & $+/-$ & - & - & - \\
\hline F14 & + & $+/-$ & + & + & $+/-$ & + & $+/-$ & $+/-$ & - & & - & - \\
\hline
\end{tabular}

2 neural/neuroendocrine markers. Considering the caveats detailed for cytokeratin staining above, this indicates that the intestinal tumors of zebrafish examined here are most likely not of neuroendocrine origin. Mammalian S100 antibody has been shown to cross-react with zebrafish schwannomas (Marino et al. 2012) and also stains neural tissues in zebrafish and other fishes (Masahito et al. 1985, Bunton \& Wolfe 1996, Manso et al. 1997, Bunton 2000, Sakamoto \& White 2002, Marino et al. 2007). However, it did not cross-react in a dysembryoplastic neuroepithelial tumor of zebrafish that we recently described (Peterson et al. 2013).

Chromogranin A rabbit antibodies have not previously been investigated with the zebrafish model. Here, we observed specific staining of neural tissues, particularly in nerve ganglia and the pituitary gland. In contrast, none of the neoplasms exhibited staining for this antibody. By immunoblot analysis, chromogranin A expressed a distinct band at $\sim 44 \mathrm{kDa}$, which was lower than the predicted zebrafish chromogranin A molecular weight, based on sequence data (Montero-Hadjadje et al. 2008) but similar to that observed in brown bullhead Ameiurus nebulosus (Bunton 2000).

Western blots for S100 and chromogranin A produced only weak bands, and only the former appeared at the expected size. The other 3 antibodies consistently reacted to produce protein bands 11 to $16 \mathrm{kDa}$ below their expected molecular weights against the zebrafish tissue (Conrad et al. 1998, García et al. 2005, Germanà et al. 2007, Montero-Hadjadje et al. 2008). For most zebrafish proteins, only molecular weight estimations based on amino acid sequence are available. However, additional support for the immunoblot findings might be obtainable using PCR and genetic analysis of the zebrafish proteins.

Antibodies directed at mammalian proteins are not optimized for use on zebrafish tissue sections, and even certain antibodies generated against other teleost fish have shown markedly reduced affinity for zebrafish antigens (García et al. 2005). Moreover, the few zebrafish-specific antibodies that have been developed to date have not been optimized or appropriately validated (Feitsma \& Cuppen 2008). Nevertheless, given the morphology and location of the neoplastic cells, and our results with immunohistochemistry, we conclude that most, if not all, of the commonly observed intestinal tumors seen in zebrafish are derived from epithelial cells.

Acknowledgements. This study was supported by departmental funds (to C.E.P.), NIH NICHD \#P01HD22486, NIH OD P40 RR012546, 2R24OD010998-11, CA090890, CA122959, and NIEHS Environmental Health Science Center \#P30 ES000210.

\section{LITERATURE CITED}

Adem C, Reynolds C, Adlakha H, Roche PC, Nascimento AG (2002) Wide spectrum screening keratin as a marker of metaplastic spindle cell carcinoma of the breast: an immunohistochemical study of 24 patients. Histopathology 40:556-562

Amatruda JF, Patton EE (2008) Genetic models of cancer in zebrafish. Int Rev Cell Mol Biol 271:1-34

Brenner B, Tang LH, Klimstra DS, Kelsen DP (2004) Smallcell carcinomas of the gastrointestinal tract: a review. J Clin Oncol 22:2730-2739

Bunton TE (1994) Tumor immunodiagnosis in fish. In: Gardner HS (ed) Compendium of the FY1990 and FY1992 research reviews for the research methods branch. Army Biomedical Research and Development Lab, Fort Detrick, MD, p 97-99

Bunton TE (2000) Brown bullhead (Ameiurus nebulosus) skin carcinogenesis. Exp Toxicol Pathol 52:209-220

Bunton TE, Wolfe MJ (1996) Reactivity of tissue-specific antigens in N-methyl-N'-nitro-N nitrosoguanidineinduced neoplasms and normal tissues from medaka (Oryzias latipes). Toxicol Pathol 24:331-338

Chu PG, Weiss LM (2002) Keratin expression in human tissues and neoplasms. Histopathology 40:403-439

Conrad M, Lemb K, Schubert T, Markl J (1998) Biochemical identification and tissue-specific expression patterns of keratins in the zebrafish Danio rerio. Cell Tissue Res 293: 195-205 
Feitsma H, Cuppen E (2008) Zebrafish as a cancer model. Mol Cancer Res 6:685-694

Ferrari L, Seregni E, Bajetta E, Martinetti A, Bombardieri E (1999) The biological characteristics of chromogranin A and its role as a circulating marker in neuroendocrine tumours. Anticancer Res 19:3415-3427

Gabbert H, Wagner R, Moll R, Gerharz CD (1985) Tumor dedifferentiation: an important step in tumor invasion. Clin Exp Metastasis 3:257-279

García DM, Bauer H, Dietz T, Schubert T, Markl J, Schaffeld $M$ (2005) Identification of keratins and analysis of their expression in carp and goldfish: comparison with the zebrafish and trout keratin catalog. Cell Tissue Res 322: $245-256$

Germanà A, Paruta S, Germanà GP, Ochoa-Erena FJ, Montalbano G, Cobo J, Vega JA (2007) Differential distribution of S100 protein and calretinin in mechanosensory and chemosensory cells of adult zebrafish (Danio rerio). Brain Res 1162:48-55

Giandomenico V (2010) Molecular pathology of gastrointestinal neuroendocrine tumours - selected topics. Diagn Histopathol 16:243-250

> Jirásek T, Mandys V (2003) Different patterns of chromogranin A and Leu-7 (CD57) expression in gastrointestinal carcinoids: immunohistochemical and confocal scanning microscopy study. Neoplasma 50:1-7

Kapoor BG, Khanna B (2004) Ichthyology handbook. Narosa Publishing House, New Delhi

Klöppel G, Anlauf M (2005) Epidemiology, tumour biology and histopathological classification of neuroendocrine tumours of the gastrointestinal tract. Best Pract Res Clin Gastroenterol 19:507-517

Lingeman CH, Garner FM (1972) Comparative study of intestinal adenocarcinomas of animals and man. J Natl Cancer Inst 48:325-346

Liu S, Leach SD (2011) Zebrafish models for cancer. Annu Rev Pathol 6:71-93

Manso MJ, Becerra M, Becerra M, Anadón R (1997) Expression of a low-molecular weight (10 kDa) calcium binding protein in glial cells of the brain of the trout (Teleostei). Anat Embryol (Berl) 196:403-416

Marino F, Germanà A, Bambir S, Helgason S, De Vico G, Macrì B (2007) Calretinin and S-100 expression in goldfish, Carassius auratus (L.), schwannoma. J Fish Dis 30: 251-253

Editorial responsibility: Thomas Lang,

Cuxhaven, Germany
Marino F, Lanteri G, Rapisarda G, Perillo A, Macrì B (2012) Spontaneous schwannoma in zebrafish, Danio rerio (Hamilton). J Fish Dis 35:239-242

Masahito P, Ishikawa T, Yanagisawa A, Sugano H, Ikeda K (1985) Neurogenic tumors in Coho salmon (Oncorhynchus kisutch) reared in well water in Japan. J Natl Cancer Inst 75:779-790

Miettinen M, Lasota J (2001) Gastrointestinal stromal tumours - definition, clinical, histological, immunohistochemical, and molecular genetic features and differential diagnosis. Virchows Arch 438:1-12

Modlin IM, Oberg J, Chung DC, Jensen RT and others (2008) Gastroenteropancreatic neuroendocrine tumours. Lancet Oncol 9:61-72

Montero-Hadjadje M, Vaingankar S, Elias S, Tostivint H, Mahata SK, Anouar Y (2008) Chromogranins A and $\mathrm{B}$ and secretogranin II: evolutionary and functional aspects. Acta Physiol (Oxf) 192:309-324

> Paquette CE, Kent ML, Buchner C, Tanguay TL, Guillemin K, Mason TJ, Peterson TS (2013) A retrospective study of the prevalence and classification of intestinal neoplasia in zebrafish (Danio rerio). Zebrafish 10:228-236

> Peterson TS, Heidel JR, Murray KN, Sanders JL, Anderson WI, Kent ML (2013) Dysembryoplastic neuroepithelial tumor in a zebrafish (Danio rerio). J Comp Pathol 148: $220-224$

Sakamoto K, White M (2002) Dermal melanoma with schwannoma-like differentiation in a brown bullhead catfish (Ictalurus nebulosus). J Vet Diagn Invest 14: $247-250$

> Sidhu GS (1979) The endodermal origin of digestive and respiratory tract APUD cells. Histopathologic evidence and a review of the literature. Am J Pathol 96:5-20

> Singh VK, Cheng JF (1996) Immunoreactive S100 proteins of blood immunocytes and brain cells. J Neuroimmunol 64:135-139

Spitsbergen JM, Buhler DR, Peterson TS (2012) Neoplasia and neoplasm-associated lesions in laboratory colonies of zebrafish emphasizing key influences of diet and aquaculture system design. ILAR J 53:114-125

Woodcock-Mitchell J, Eichner R, Nelson WG, Sun TT (1982) Immunolocalization of keratin polypeptides in human epidermis using monoclonal antibodies. J Cell Biol 95: 580-588

Submitted: May 21, 2015; Accepted: September 7, 2015 Proofs received from author(s): October 11, 2015 